\title{
Permanence of a Gilpin-Ayala predator-prey system with time-dependent delay
}

Hongying $\mathrm{Lu}^{1 *}$ and Gang $\mathrm{Yu}^{1,2}$

\section{*Correspondence:}

hongyinglu543@163.com

'School of Mathematics and

Quantitative Economics, Dongbei

University of Finance and

Economics, Jianshan street, Dalian,

China

Full list of author information is

available at the end of the article

\begin{abstract}
In this paper, a Gilpin-Ayala predator-prey model with time-dependent delay with $m$ preys and $(n-m)$ predators is studied, which can be seen as a modification of the traditional Lotka-Volterra competition predator-prey system model. Two sets of sufficient conditions on the permanence of the system are obtained. One set is delay dependent, while the other set is delay independent.
\end{abstract}

Keywords: permanence; non-autonomous system; Gilpin-Ayala predator-prey model; time-dependent delay

\section{Introduction}

Predator-prey phenomena are always interesting topics in both ecology and mathematical ecology, which attract a lot of attention due to its universal existence and importance [1]. Since Volterra [2] proposed a differential equation model to successfully explain the ecological phenomenon of Finme Fish Harbor in 1926, and Lotka [3] also derived the model to describe a hypothetical chemical reaction in which the chemical concentrations oscillate in 1925, the famous Lotka-Volterra equations were accepted by many experts. The classical Lotka-Volterra predator-prey model is a rudimentary model of mathematical ecology which can be expressed as follows:

$$
\begin{aligned}
& \dot{x}(t)=x(t)(b-a y(t)), \\
& \dot{y}(t)=y(t)(-d+c x(t)),
\end{aligned}
$$

where $x(t)$ is the density of the prey species at time $t, y(t)$ is the density of the predator species at time $t . b$ is the intrinsic growth rate of the prey, $a$ is the per-capita rate of predation of the predator, $d$ is the death rate of the predator, $c$ denotes the product of the per-capita rate of predation and the rate of conversing prey into predator.

Since then the Lotka-Volterra equation and its various generalized forms have been frequently used to describe the population dynamics with predator-prey relations and a lot of extensive research results have already been obtained and one has seen great progress [4-25]. However, regardless of this fact, the Lotka-Volterra equation has a property which is considered as a disadvantage and that is the linearity of this model (i.e. the rate of change in the size of each species is a linear function of the sizes of the interacting species). Particularly, in 1973, Gilpin and Ayala [26] claimed that a little more complicated model was 
needed in order to obtain more realistic solutions and proposed the following model:

$$
\dot{x}_{i}(t)=r_{i} x_{i}(t)\left[1-\left(\frac{x_{i}(t)}{K_{i}}\right)^{\theta_{i}}-\sum_{j=1, j \neq i}^{n} a_{i j}(t) \frac{x_{j}(t)}{K_{j}}\right], \quad i=1,2, \ldots, n,
$$

where $x_{i}(t)$ is the population density of species $i$ at time $t, r_{i}$ is the intrinsic exponential growth rate of species $i, K_{i}$ is the environment carrying capacity of species $i$ in the absence of competition, $\theta_{i}$ provides a nonlinear measure of intraspecific interference, and $a_{i j}(i \neq j)$ provides a measure of interspecific competition. So, $\mathrm{Li}$ and $\mathrm{Lu}$ [5] introduced the following more complicated non-autonomous prey-competition model:

$$
\begin{aligned}
& \dot{x}_{i}(t)=x_{i}(t)\left[r_{i}(t)-\sum_{j=1}^{n} a_{i j}(t) x_{j}^{\alpha_{i j}}(t)\right], \quad i=1,2, \ldots, m, \\
& \dot{x}_{i}(t)=x_{i}(t)\left[-r_{i}(t)+\sum_{j=1}^{m} a_{i j}(t) x_{j}^{\alpha_{i j}}(t)-\sum_{j=m+1}^{n} a_{i j}(t) x_{j}^{\alpha_{i j}}(t)\right], \quad i=m+1, \ldots, n,
\end{aligned}
$$

they obtained sufficient conditions for the existence of a unique globally attractive periodic solution of system (1.3). For more work in this direction, one could refer to [5, 14-16] and the references cited therein.

Furthermore, delay due to negative feedback is a common example, because the process of a reproduction of a population is not instantaneous. The effect of these kinds of delays on the asymptotic behavior of populations has been studied by a number of authors (see, for example, [11, 15, 20, 22-25]). Chen et al. [6] further incorporated time delays in the model (1.3) and they proposed the following model:

$$
\left\{\begin{aligned}
\dot{x}_{i}(t)= & x_{i}(t)\left[r_{i}(t)-\sum_{j=1}^{n} a_{i j}(t) x_{j}^{\alpha_{i j}}(t)-\sum_{j=1}^{n} b_{i j}(t) x_{j}^{\beta_{i j}}\left(t-\tau_{i j}(t)\right)\right], \quad i=1,2, \ldots, m, \\
\dot{x}_{i}(t)= & x_{i}(t)\left[-r_{i}(t)+\sum_{j=1}^{m} a_{i j}(t) x_{j}^{\alpha_{i j}}(t)+\sum_{j=1}^{m} b_{i j}(t) x_{j}^{\beta_{i j}}\left(t-\tau_{i j}(t)\right)\right. \\
& \left.-\sum_{j=m+1}^{n} a_{i j}(t) x_{j}^{\alpha_{i j}}(t)-\sum_{j=m+1}^{n} b_{i j}(t) x_{j}^{\beta_{i j}}\left(t-\tau_{i j}(t)\right)\right], \quad i=m+1, \ldots, n,
\end{aligned}\right.
$$

with the initial conditions

$$
x_{i}(s)=\Phi_{i}(s) \geq 0, \quad s \in[-\tau, 0] ; \quad \Phi_{i}(0)>0 ;
$$

where $\tau=\max _{1 \leq i, j \leq n}\left\{\sup _{t \in[0,+\infty)}\left\{\tau_{i j}(t)\right\}\right\} . x_{i}(t), i=1,2, \ldots, m$, are the densities of the prey species $i$ at time $t, x_{i}(t), i=m+1, \ldots, n$ are the densities of the predator species $X_{i}$ at time $t ; \alpha_{i j}, \beta_{i j}$ are all positive constants and represent nonlinear measures of interspecific or intraspecific interference. $r_{i}(t), i=1, \ldots, m$, and $r_{i}(t), i=m+1, \ldots, n$, are the intrinsic and death rates at time $t, a_{i j}(t)$ and $b_{i j}(t)$ represent the effects of the interspecific $(i \neq j)$ and the intraspecific $(i=j)$ interaction at time $t$; the terms $b_{i j}(t) x_{j}^{\beta_{i j}}\left(t-\tau_{i j}(t)\right)$ represent the negative feedback crowding. By using the Gaines and Mawhins continuation theorem of coincidence degree theory and by constructing an appropriate Lyapunov functional, they obtained a set of sufficient conditions which guarantee the existence and global attractivity of positive periodic solutions of the system (1.4).

As we know, a more important theme that interested mathematicians as well as biologists is whether all species in a multi-species community would survive in the long run, 
that is, whether the ecosystems are permanent (see, for example, $[1,5,7-13,15,18-25])$. Huang [7] studied the permanence of the system (1.4). By using comparison theory and a differential inequality, two sets of sufficient conditions which guarantee the permanence of the system (1.4) are obtained. Their results supplement the main results of Chen et al. [6]. In this paper, we shall also study the permanence of the system (1.4) by using comparison theory, and get the same results as [7] do, under weaker conditions.

In this paper, we also explore the system (1.4). Throughout this paper, we always assume that for all $i, j=1,2, \ldots, n$ :

$\left(\mathrm{H}_{1}\right)$ The bounded functions $r_{i}(t), a_{i j}(t), b_{i j}(t), \tau_{i j}(t)$ are all nonnegative and continuous for all $t \in R$ and $a_{i i}^{l} \geq 0, b_{i i}^{l} \geq 0, a_{i i}^{l}+b_{i i}^{l}>0$. Here, for any bounded function $f(t)$, $f^{u}=\lim _{t \rightarrow \infty} \sup f(t), f^{l}=\lim _{t \rightarrow \infty} \inf f(t) ;$

$\left(\mathrm{H}_{2}\right) \alpha_{i j}, \beta_{i j}$ are all positive constants.

This paper is aimed at obtaining, by developing the analytical technique of [9-11], two sets of sufficient conditions which guarantee the permanence of the system (1.4). One set is delay dependent, while the other set is delay independent. Our results improve on Theorems 2.1 and 2.2 of [7]. Moreover, we state and prove the main results in the next section and present a brief conclusion. For more background and biological adjustments of system (1.4), see $[4,6,7,14-17,19-21]$ and the references cited therein.

\section{Main results}

In this section, we present two sets of sufficient conditions for the permanence of system (1.4). We denote by $R_{+}^{n}=\left\{\left(x_{1}, \ldots, x_{n}\right) \in R^{n} \mid x_{i} \geq 0, i=1,2, \ldots, n\right\}$ the nonnegative cone and by Int $R_{+}^{n}=\left\{\left(x_{1}, \ldots, x_{n}\right) \in R^{n} \mid x_{i}>0, i=1,2, \ldots, n\right\}$ the positive cone. For ecological reasons, we consider system (1.4), only in $\operatorname{Int} R_{+}^{n}$.

Definition 2.1 System (1.4) is said to be permanent, if there are positive constants $m$ and $M$, such that for each positive solution $\left(x_{1}(t), \ldots, x_{n}(t)\right)^{T}$ of system (1.4) satisfies

$$
m \leq \lim _{t \rightarrow+\infty} \inf x_{i}(t) \leq \lim _{t \rightarrow+\infty} \sup x_{i}(t) \leq M, \quad i=1,2, \ldots, n .
$$

It is easy to prove the following lemma.

Lemma 2.1 The positive cone is invariant with respect to system (1.4).

For system (1.4), we will consider two cases, $a_{i i}^{l}>0, b_{i i}^{l} \geq 0$ and $a_{i i}^{l} \geq 0, b_{i i}^{l}>0$, respectively, then we obtain Theorems 2.1 and 2.2.

For convenience, we introduce the following notations.

$$
\begin{aligned}
\text { For } i=1, \ldots, m & \begin{aligned}
M_{i} & =\left(\frac{r_{i}^{u}}{a_{i i}^{l}}\right)^{\frac{1}{\alpha_{i i}}} ; \\
m_{i} & =\left(\frac{r_{i}^{l}-\sum_{j=1, j \neq i}^{n} a_{i j}^{u} M_{j}^{\alpha_{i j}}-\sum_{j=1}^{n} b_{i j}^{u} M_{j}^{\beta_{i j}}}{a_{i i}^{u}}\right)^{\frac{1}{\alpha_{i i}}} ;
\end{aligned}
\end{aligned}
$$


for $i=m+1, \ldots, n$

$$
\begin{aligned}
& M_{i}=\left(\frac{-r_{i}^{l}+\sum_{j=1}^{m} a_{i j}^{u} M_{j}^{\alpha_{i j}}+\sum_{j=1}^{m} b_{i j}^{u} M_{j}^{\beta_{i j}}}{a_{i i}^{l}}\right)^{\frac{1}{\alpha_{i i}}} ; \\
& m_{i}=\left(\frac{\Delta_{i}}{a_{i i}^{u}}\right)^{\frac{1}{\alpha_{i i}}},
\end{aligned}
$$

where

$$
\Delta_{i}=-r_{i}^{u}+\sum_{j=1}^{m} a_{i j}^{l} m_{j}^{\alpha_{i j}}+\sum_{j=1}^{n} b_{i j}^{l} m_{j}^{\beta_{i j}}-\sum_{j=m+1, j \neq i}^{n} a_{i j}^{u} M_{j}^{\alpha_{i j}}-\sum_{j=m+1}^{n} b_{i j}^{u} M_{j}^{\beta_{i j}} .
$$

Theorem 2.1 Assume that system (1.4) satisfies $a_{i i}^{l}>0$ and the following.

$\left(\mathrm{H}_{3}\right) r_{i}^{l}>\sum_{j=1, j \neq i}^{n} a_{i j}^{u} M_{j}^{\alpha_{i j}}+\sum_{j=1}^{n} b_{i j}^{u} M_{j}^{\beta_{i j}}, i=1,2, \ldots, m$,

$\left(\mathrm{H}_{4}\right) \quad \Delta_{i}>0, i=m+1, \ldots, n$.

Then system (1.4) is permanent.

Proof Let $x(t)=\left(x_{1}(t), \ldots, x_{n}(t)\right)^{T}$ be any positive solution of system (1.4) with initial condition (1.5). For $i=1,2, \ldots, m$, it follows from system (1.4) that

$$
\dot{x}_{i}(t) \leq x_{i}(t)\left(r_{i}(t)-a_{i i}(t) x_{i}^{\alpha i i}(t)\right)
$$

thus

$$
\frac{d\left(x_{i}^{\alpha_{i i}}(t)\right)}{d t} \leq \alpha_{i i} x_{i}^{\alpha_{i i}}(t)\left(r_{i}(t)-a_{i i}(t) x_{i}^{\alpha_{i i}}(t)\right)
$$

Let $u_{i}(t)=x_{i}^{\alpha_{i i}}(t)$, we have

$$
\dot{u}_{i}(t) \leq \alpha_{i i} u_{i}(t)\left(r_{i}(t)-a_{i i}(t) u_{i}(t)\right) \leq \alpha_{i i} u_{i}(t)\left(r_{i}^{u}-a_{i i}^{l} u_{i}(t)\right)
$$

By using the comparability theorem, we obtain

$$
\lim _{t \rightarrow+\infty} \sup u_{i}(t) \leq \frac{r_{i}^{u}}{a_{i i}^{l}}
$$

so it immediately follows that

$$
\lim _{t \rightarrow+\infty} \sup x_{i}(t) \leq\left(\frac{r_{i}^{u}}{a_{i i}^{l}}\right)^{\frac{1}{\alpha_{i i}}}:=M_{i}, \quad i=1,2, \ldots, m
$$

For any $\varepsilon>0$ small enough, it follows from (2.5) that there exists large enough $T_{1}$ such that for all $i=1,2, \ldots, m$ and $t \geq T_{1}$

$$
x_{i}(t) \leq M_{i}+\varepsilon \text {. }
$$


For $i=m+1, \ldots, n$ and $t \geq T_{1}+\tau,(2.6)$ combining with the $i$ th equation of system (1.4) leads to

$$
\dot{x}_{i}(t) \leq x_{i}(t)\left(-r_{i}(t)+\sum_{j=1}^{m} a_{i j}(t)\left(M_{j}+\varepsilon\right)^{\alpha_{i j}}+\sum_{j=1}^{m} b_{i j}(t)\left(M_{j}+\varepsilon\right)^{\beta_{i j}}-a_{i i}(t) x_{i}^{\alpha_{i i}}(t)\right),
$$

thus by a similar argument, we can verify that

$$
\lim _{t \rightarrow+\infty} \sup x_{i}(t) \leq\left(\frac{-r_{i}^{l}+\sum_{j=1}^{m} a_{i j}^{u} M_{j}^{\alpha_{i j}}+\sum_{j=1}^{m} b_{i j}^{u} M_{j}^{\beta_{i j}}}{a_{i i}^{l}}\right)^{\frac{1}{\alpha_{i i}}}:=M_{i}, \quad i=m+1, \ldots, n
$$

From the condition $\left(\mathrm{H}_{3}\right)$ of Theorem 2.1, we could choose $\varepsilon>0$ small enough such that

$$
r_{i}^{l}>\sum_{j=1, j \neq i}^{n} a_{i j}^{u}\left(M_{j}+\varepsilon\right)^{\alpha_{i j}}+\sum_{j=1}^{n} b_{i j}^{u}\left(M_{j}+\varepsilon\right)^{\beta_{i j}}
$$

thus, for $\varepsilon>0$ satisfies (2.9), from (2.5) and (2.8), we know that there exists $T_{2}>T_{1}+\tau$ such that for all $i=1,2, \ldots, n$ and $t \geq T_{2}$

$$
x_{i}(t) \leq M_{i}+\varepsilon
$$

For $i=1, \ldots, m$ and $t \geq T_{2}+\tau$, by applying (2.10), from the $i$ th equation of system (1.4), we have

$$
\dot{x}_{i}(t) \geq x_{i}(t)\left(r_{i}(t)-\sum_{j=1, j \neq i}^{n} a_{i j}(t)\left(M_{j}+\varepsilon\right)^{\alpha_{i j}}-\sum_{j=1}^{n} b_{i j}(t)\left(M_{j}+\varepsilon\right)^{\beta_{i j}}-a_{i i}(t) x_{i}^{\alpha_{i i}}(t)\right),
$$

thus

$$
\begin{aligned}
\frac{d\left(x_{i}^{\alpha_{i i}}(t)\right)}{d t} \geq & \alpha_{i i} x_{i}^{\alpha_{i i}}(t)\left(r_{i}(t)-\sum_{j=1, j \neq i}^{n} a_{i j}(t)\left(M_{j}+\varepsilon\right)^{\alpha_{i j}}\right. \\
& \left.-\sum_{j=1}^{n} b_{i j}(t)\left(M_{j}+\varepsilon\right)^{\beta_{i j}}-a_{i i}(t) x_{i}^{\alpha_{i i}}(t)\right),
\end{aligned}
$$

let $u_{i}(t)=x_{i}^{\alpha_{i i}}(t)$, we get

$$
\begin{aligned}
\dot{u}_{i}(t) & \geq \alpha_{i i} u_{i}(t)\left(r_{i}(t)-\sum_{j=1, j \neq i}^{n} a_{i j}(t)\left(M_{j}+\varepsilon\right)^{\alpha_{i j}}-\sum_{j=1}^{n} b_{i j}(t)\left(M_{j}+\varepsilon\right)^{\beta_{i j}}-a_{i i}(t) u_{i}(t)\right) \\
& \geq \alpha_{i i} u_{i}(t)\left(r_{i}^{l}-\sum_{j=1, j \neq i}^{n} a_{i j}^{u}\left(M_{j}+\varepsilon\right)^{\alpha_{i j}}-\sum_{j=1}^{n} b_{i j}^{u}\left(M_{j}+\varepsilon\right)^{\beta_{i j}}-a_{i i}^{u} u_{i}(t)\right) .
\end{aligned}
$$

According to the comparability theorem, we have

$$
\lim _{t \rightarrow+\infty} \inf u_{i}(t) \geq \frac{r_{i}^{l}-\sum_{j=1, j \neq i}^{n} a_{i j}^{u}\left(M_{j}+\varepsilon\right)^{\alpha_{i j}}-\sum_{j=1}^{n} b_{i j}^{u}\left(M_{j}+\varepsilon\right)^{\beta_{i j}}}{a_{i i}^{u}}
$$


setting $\varepsilon \rightarrow 0$ in above inequality, we have

$$
\lim _{t \rightarrow+\infty} \inf u_{i}(t) \geq \frac{r_{i}^{l}-\sum_{j=1, j \neq i}^{n} a_{i j}^{u} M_{j}^{\alpha_{i j}}-\sum_{j=1}^{n} b_{i j}^{u} M_{j}^{\beta_{i j}}}{a_{i i}^{u}},
$$

therefore

$$
\lim _{t \rightarrow+\infty} \inf x_{i}(t) \geq\left(\frac{r_{i}^{l}-\sum_{j=1, j \neq i}^{n} a_{i j}^{u} M_{j}^{\alpha_{i j}}-\sum_{j=1}^{n} b_{i j}^{u} M_{j}^{\beta_{i j}}}{a_{i i}^{u}}\right)^{\frac{1}{\alpha_{i i}}}:=m_{i}, \quad i=1,2, \ldots, m .
$$

From the condition $\left(\mathrm{H}_{4}\right)$ of Theorem 2.1, we could choose $\varepsilon>0$ small enough such that

$$
\begin{aligned}
-r_{i}^{u} & +\sum_{j=1}^{m} a_{i j}^{l}\left(m_{j}-\varepsilon\right)^{\alpha_{i j}}+\sum_{j=1}^{m} b_{i j}^{l}\left(m_{j}-\varepsilon\right)^{\beta_{i j}} \\
& -\sum_{j=m+1, j \neq i}^{n} a_{i j}^{u}\left(M_{j}+\varepsilon\right)^{\alpha_{i j}}-\sum_{j=m+1}^{n} b_{i j}^{u}\left(M_{j}+\varepsilon\right)^{\beta_{i j}} \geq 0,
\end{aligned}
$$

thus, for $\varepsilon>0$ it satisfies (2.16), and it follows from (2.15) that there exists large enough $T_{3}>T_{2}+\tau$ such that for all $i=1, \ldots, m$ and $t \geq T_{3}$

$$
x_{i}(t) \geq m_{i}-\varepsilon,
$$

thus, for $i=m+1, \ldots, n$ and $t \geq T_{3}+\tau,(2.10)$ and (2.17) combining with the $i$ th equation of system (1.4) leads to

$$
\begin{aligned}
\dot{x}_{i}(t) \geq & x_{i}(t)\left(-r_{i}(t)+\sum_{j=1}^{m} a_{i j}(t)\left(m_{j}-\varepsilon\right)^{\alpha_{i j}}+\sum_{j=1}^{m} b_{i j}(t)\left(m_{j}-\varepsilon\right)^{\beta_{i j}}\right. \\
& \left.-\sum_{j=m+1, j \neq i}^{n} a_{i j}(t)\left(M_{j}+\varepsilon\right)^{\alpha_{i j}}-\sum_{j=m+1}^{n} b_{i j}(t)\left(M_{j}+\varepsilon\right)^{\beta_{i j}}-a_{i i}(t) x_{i}^{\alpha_{i i}}(t)\right),
\end{aligned}
$$

by using (2.18), similarly, we obtain

$$
\lim _{t \rightarrow+\infty} \inf x_{i}(t) \geq\left(\frac{\Delta_{i}}{a_{i i}^{u}}\right)^{\frac{1}{\alpha_{i i}}}:=m_{i}, \quad i=m+1, \ldots, n,
$$

where

$$
\Delta_{i}=-r_{i}^{u}+\sum_{j=1}^{m} a_{i j}^{l} m_{j}^{\alpha_{i j}}+\sum_{j=1}^{n} b_{i j}^{l} m_{j}^{\beta_{i j}}-\sum_{j=m+1, j \neq i}^{n} a_{i j}^{u} M_{j}^{\alpha_{i j}}-\sum_{j=m+1}^{n} b_{i j}^{u} M_{j}^{\beta_{i j}} .
$$

Take $M=\max _{1 \leq i \leq n}\left\{M_{i}\right\}, m=\min _{1 \leq i \leq n}\left\{m_{i}\right\}$, we have

$$
m \leq \liminf _{t \rightarrow+\infty} x_{i}(t) \leq \limsup _{t \rightarrow+\infty} x_{i}(t) \leq M, \quad i=1,2, \ldots, n
$$

This ends the proof of Theorem 2.1. 
Denote for $i=1, \ldots, m$

$$
\begin{aligned}
& \bar{M}_{i}=\left(\frac{r_{i}^{u}}{b_{i i}^{l}}\right)^{\frac{1}{\beta_{i i}}} \exp \left[r_{i}^{u} \tau\right] ; \\
& \bar{m}_{i}=\min _{1 \leq i \leq n}\left\{\left(\frac{r_{i}^{l}}{2 b_{i}^{u}}\right)^{\frac{1}{\beta_{i i}}}, l_{i}^{\frac{1}{\beta_{i i}}}\right\},
\end{aligned}
$$

where

$$
\begin{aligned}
& \tau=\max _{1 \leq i, j \leq n}\left\{\sup _{t \in[0,+\infty)}\left\{\tau_{i j}(t)\right\}\right\} ; \\
& l_{i} \leq\left\{\left(r_{i}^{l}-\sum_{j=1}^{n} a_{i j}^{u} \bar{M}_{j}^{\alpha_{i j}}-\sum_{j=1, j \neq i}^{n} b_{i j}^{u} \bar{M}_{j}^{\beta_{i j}}\right) / b_{i i}^{u}, i=1, \ldots, m\right\} ;
\end{aligned}
$$

for $i=m+1, \ldots, n$

$$
\begin{aligned}
& \bar{M}_{i}=\left(\frac{\lambda_{i}}{b_{i i}^{l}}\right)^{\frac{1}{\beta_{i i}}} \exp \left[\lambda_{i} \tau\right] ; \\
& \bar{m}_{i}=\min _{1 \leq i \leq n}\left\{\left(\frac{-r_{i}^{u}+\sum_{j=1}^{m} a_{i j}^{l} m_{j}^{\alpha_{i j}}+\sum_{j=1}^{m} b_{i j}^{l} m_{j}^{\beta_{i j}}}{2 b_{i}^{u}}\right)^{\frac{1}{\beta_{i i}}}, l_{i}^{\frac{1}{\beta_{i i}}}\right\},
\end{aligned}
$$

where

$$
\begin{aligned}
& \lambda_{i}=-r_{i}^{l}+\sum_{j=1}^{m} a_{i j}^{u} \bar{M}_{j}^{\alpha_{i j}}+\sum_{j=1}^{m} b_{i j}^{u} \bar{M}_{j}^{\beta_{i j}} ; \\
& l_{i} \leq\left\{\nabla_{i} / b_{i i}^{u}, i=m+1, \ldots, n\right\} ; \\
& \nabla_{i}=-r_{i}^{u}+\sum_{j=1}^{m} a_{i j}^{l} m_{j}^{\alpha_{i j}}+\sum_{j=1}^{n} b_{i j}^{l} m_{j}^{\beta_{i j}}-\sum_{j=m+1}^{n} a_{i j}^{u} M_{j}^{\alpha_{i j}}-\sum_{j=m+1, j \neq i}^{n} b_{i j}^{u} M_{j}^{\beta_{i j}} .
\end{aligned}
$$

Theorem 2.2 Assume that system (1.4) satisfies $b_{i i}^{l}>0$ and the following.

$\left(\mathrm{H}_{5}\right) r_{i}^{l}>\sum_{j=1}^{n} a_{i j}^{u} \bar{M}_{j}^{\alpha_{i j}}+\sum_{j=1, j \neq i}^{n} b_{i j}^{u} \bar{M}_{j}^{\beta_{i j}}, i=1,2, \ldots, m$,

$\left(\mathrm{H}_{6}\right) \nabla_{i}>0, i=m+1, \ldots, n$.

Then system (1.4) is permanent.

Proof Let $x(t)=\left(x_{1}(t), \ldots, x_{n}(t)\right)^{T}$ be any positive solution of system (1.4) with initial condition (1.5), for $i=1,2, \ldots, m$, it follows from system (1.4) that

$$
\dot{x}_{i}(t) \leq x_{i}(t)\left(r_{i}(t)-b_{i i}(t) x_{i}^{\beta_{i i}}\left(t-\tau_{i i}(t)\right)\right) \text {, }
$$

thus

$$
\frac{d\left(x_{i}^{\beta_{i i}}(t)\right)}{d t} \leq \beta_{i i} x_{i}^{\beta_{i i}}(t)\left(r_{i}(t)-b_{i i}(t) x_{i}^{\beta_{i i}}\left(t-\tau_{i i}(t)\right)\right) .
$$


Let $u_{i}(t)=x_{i}^{\beta_{i i}}(t)$, we have

$$
\begin{aligned}
\dot{u}_{i}(t) & \leq \beta_{i i} u_{i}(t)\left(r_{i}(t)-b_{i i}(t) u_{i}\left(t-\tau_{i i}(t)\right)\right) \\
& \leq \beta_{i i} u_{i}(t)\left(r_{i}^{u}-b_{i i}^{l} u_{i}\left(t-\tau_{i i}(t)\right)\right) .
\end{aligned}
$$

Take $\tilde{M}_{i}=\frac{r_{i}^{u}}{b_{i i}^{l}}\left(1+h_{i}\right)$, where $0<h_{i}<\exp \left[r_{i}^{u} \tau\right]-1, \tau=\max _{1 \leq i, j \leq n}\left\{\sup _{t \in[0,+\infty)}\left\{\tau_{i j}(t)\right\}\right\}$. Firstly, suppose $u_{i}(t)$ is not oscillatory about $\tilde{M}_{i}$. That is, there exists a $T_{1}^{*}>0$, for $t>T_{1}^{*}$ such that

$$
u_{i}(t)<\tilde{M}_{i}
$$

or

$$
u_{i}(t)>\tilde{M}_{i}
$$

If (2.23) holds, then our aim is reached. Suppose (2.24) holds, then for $t \geq T_{1}^{*}+\tau$, we obtain

$$
\dot{u}_{i}(t)<-\beta_{i i} h_{i} r_{i}^{u} u_{i}(t)
$$

thus $u_{i}(t)<u_{i}(0) \exp \left[-\beta_{i i} h_{i} r_{i}^{u} t\right] \rightarrow 0$, as $t \rightarrow+\infty$, which is in contradiction with (2.24). Hence there must exist $\tilde{T}_{1}>T_{1}^{*}+\tau$ such that $u_{i}(t)<\tilde{M}_{i}$ for $t>\tilde{T}_{1}$. Secondly now assume that $u_{i}(t)$ is oscillatory about $\tilde{M}_{i}$ for $t \geq T_{1}^{*}$, that is, there exists a time sequence $\left\{t_{n}\right\}$ such that $\tau<t_{i}<t_{2}<\cdots<t_{n}<\cdots$ is a sequence of zeros of $u_{i}\left(t_{n}\right)-\tilde{M}_{i}$ with $\lim _{n \rightarrow \infty} t_{n}=+\infty$ and $u_{i}\left(t_{n}\right)=\tilde{M}_{i}$. Set $\bar{t}_{n}$ to be a point where $u_{i}(t)$ attends its maximum in $\left(t_{n}, t_{n+1}\right)$. Thus we get $u_{i}\left(\bar{t}_{n}\right) \geq u_{i}\left(t_{n}\right)=\tilde{M}_{i}$. Then it is easy to see from (2.22) that

$$
0=\left.\dot{u}_{i}(t)\right|_{t=\bar{t}_{n}} \leq \beta_{i i} u_{i}\left(\bar{t}_{n}\right)\left(r_{i}^{u}-b_{i i}^{l} u_{i}\left(\bar{t}_{n}-\tau_{i i}\left(\bar{t}_{n}\right)\right)\right),
$$

which implies that

$$
u_{i}\left(\bar{t}_{n}-\tau_{i i}\left(\bar{t}_{n}\right)\right) \leq \frac{r_{i}^{u}}{b_{i i}^{l}}
$$

Integrating the both sides of (2.22) from $\bar{t}_{n}-\tau_{i i}\left(\bar{t}_{n}\right)$ to $\bar{t}_{n}$, it follows that

$$
\ln \frac{u_{i}\left(\bar{t}_{n}\right)}{u_{i}\left(\bar{t}_{n}-\tau_{i i}\left(\bar{t}_{n}\right)\right)} \leq \int_{\bar{t}_{n}-\tau_{i i}\left(\bar{t}_{n}\right)}^{\bar{t}_{n}} \beta_{i i}\left(r_{i}^{u}-b_{i i}^{l} u_{i}\left(t-\tau_{i i}(t)\right)\right) d t \leq \beta_{i i} r_{i}^{u} \tau_{i i}\left(\bar{t}_{n}\right) .
$$

From (2.26) and (2.27) we get

$$
u_{i}\left(\bar{t}_{n}\right) \leq \frac{r_{i}^{u}}{b_{i i}^{l}} \exp \left[\beta_{i i} r_{i}^{u} \tau\right]:=L_{i}
$$

Since $u_{i}\left(\bar{t}_{n}\right)$ is an arbitrary local maximum of $u_{i}(t)$, we can conclude that there exists a $T_{2}^{*}>0$ such that $u_{i}(t) \leq L_{i}$ for all $t \geq T_{2}^{*}$. Thus

$$
\lim _{t \rightarrow+\infty} \sup x_{i}(t) \leq\left(\frac{r_{i}^{u}}{b_{i i}^{l}}\right)^{\frac{1}{\beta_{i i}}} \exp \left[r_{i}^{u} \tau\right]:=\bar{M}_{i} .
$$


For any $\varepsilon>0$ small enough, it follows from (2.28) that there exists large enough $T_{3}^{*}>T_{2}^{*}$ such that for all $i=1,2, \ldots, m$ and $t \geq T_{3}^{*}$

$$
x_{i}(t) \leq \bar{M}_{i}+\varepsilon
$$

For $i=m+1, \ldots, n$ and $t \geq T_{3}^{*}+\tau$, (2.29) combining with the $i$ th equation of system (1.4) leads to

$$
\begin{aligned}
\dot{x}_{i}(t) \leq & x_{i}(t)\left(-r_{i}(t)+\sum_{j=1}^{m} a_{i j}(t)\left(\bar{M}_{j}+\varepsilon\right)^{\alpha_{i j}}\right. \\
& \left.+\sum_{j=1}^{m} b_{i j}(t)\left(\bar{M}_{j}+\varepsilon\right)^{\beta_{i j}}-b_{i i}(t) x_{i}^{\beta_{i i}}\left(t-\tau_{i i}(t)\right)\right),
\end{aligned}
$$

from (2.30), by a procedure similar to the discussion above, we can verify that

$$
\lim _{t \rightarrow+\infty} \sup x_{i}(t) \leq\left(\frac{\lambda_{i}}{b_{i i}^{l}}\right)^{\frac{1}{\beta_{i i}}} \exp \left[\lambda_{i} \tau\right]:=\bar{M}_{i}, \quad i=m+1, \ldots, n,
$$

where

$$
\lambda_{i}=-r_{i}^{l}+\sum_{j=1}^{m} a_{i j}^{u} \bar{M}_{j}^{\alpha_{i j}}+\sum_{j=1}^{m} b_{i j}^{u} \bar{M}_{j}^{\beta_{i j}} .
$$

From the condition $\left(\mathrm{H}_{5}\right)$ of Theorem 2.2, we could choose $\varepsilon>0$ small enough such that

$$
r_{i}^{l}>\sum_{j=1}^{n} a_{i j}^{u}\left(M_{j}+\varepsilon\right)^{\alpha_{i j}}+\sum_{j=1, j \neq i}^{n} b_{i j}^{u}\left(M_{j}+\varepsilon\right)^{\beta_{i j}},
$$

thus, for $\varepsilon>0$ it satisfies (2.32), from (2.28) and (2.31), we know that there exists $T_{4}^{*}>$ $T_{3}^{*}+\tau$ such that for all $i=1,2, \ldots, n$ and $t \geq T_{4}^{*}$

$$
x_{i}(t) \leq \bar{M}_{i}+\varepsilon .
$$

And so, for $i=1, \ldots, m$ and $t \geq T_{4}^{*}+\tau$, by applying (2.33), from the $i$ th equation of system (1.4), one has

$$
\dot{x}_{i}(t) \geq x_{i}(t)\left(r_{i}(t)-\sum_{j=1}^{n} a_{i j}(t)\left(\bar{M}_{j}+\varepsilon\right)^{\alpha_{i j}}-\sum_{j=1}^{n} b_{i j}(t)\left(\bar{M}_{j}+\varepsilon\right)^{\beta_{i j}}\right)
$$

and

$$
\begin{aligned}
\dot{x}_{i}(t) \geq & x_{i}(t)\left(r_{i}(t)-\sum_{j=1}^{n} a_{i j}(t)\left(\bar{M}_{j}+\varepsilon\right)^{\alpha_{i j}}\right. \\
& \left.-\sum_{j=1, j \neq i}^{n} b_{i j}(t)\left(\bar{M}_{j}+\varepsilon\right)^{\beta_{i j}}-b_{i i}(t) x_{i}^{\beta_{i i}}\left(t-\tau_{i i}(t)\right)\right),
\end{aligned}
$$


thus

$$
\frac{d\left(x_{i}^{\beta_{i i}}(t)\right)}{d t} \geq \beta_{i i} x_{i}^{\beta_{i i}}(t)\left(r_{i}(t)-\sum_{j=1}^{n} a_{i j}(t)\left(\bar{M}_{j}+\varepsilon\right)^{\alpha_{i j}}-\sum_{j=1}^{n} b_{i j}(t)\left(\bar{M}_{j}+\varepsilon\right)^{\beta_{i j}}\right)
$$

and

$$
\begin{aligned}
\frac{d\left(x_{i}^{\beta_{i i}}(t)\right)}{d t} \geq & \beta_{i i} x_{i}^{\beta_{i i}}(t)\left(r_{i}(t)-\sum_{j=1}^{n} a_{i j}(t)\left(\bar{M}_{j}+\varepsilon\right)^{\alpha_{i j}}\right. \\
& \left.-\sum_{j=1, j \neq i}^{n} b_{i j}(t)\left(\bar{M}_{j}+\varepsilon\right)^{\beta_{i j}}-b_{i i}(t) x_{i}^{\beta_{i i}}\left(t-\tau_{i i}(t)\right)\right) .
\end{aligned}
$$

Let $u_{i}(t)=x_{i}^{\beta_{i i}}(t)$, we have

$$
\begin{aligned}
\dot{u}_{i}(t) & \geq \beta_{i i} u_{i}(t)\left(r_{i}(t)-\sum_{j=1}^{n} a_{i j}(t)\left(\bar{M}_{j}+\varepsilon\right)^{\alpha_{i j}}-\sum_{j=1}^{n} b_{i j}(t)\left(\bar{M}_{j}+\varepsilon\right)^{\beta_{i j}}\right) \\
& \geq \beta_{i i} u_{i}(t)\left(r_{i}^{l}-\sum_{j=1}^{n} a_{i j}^{u}\left(\bar{M}_{j}+\varepsilon\right)^{\alpha_{i j}}-\sum_{j=1}^{n} b_{i j}^{u}\left(\bar{M}_{j}+\varepsilon\right)^{\beta_{i j}}\right) \\
& =\beta_{i i} u_{i}(t) \Gamma_{i \varepsilon}
\end{aligned}
$$

and

$$
\begin{aligned}
\dot{u}_{i}(t) \geq & \beta_{i i} u_{i}(t)\left(r_{i}(t)-\sum_{j=1}^{n} a_{i j}(t)\left(\bar{M}_{j}+\varepsilon\right)^{\alpha_{i j}}\right. \\
& \left.-\sum_{j=1, j \neq i}^{n} b_{i j}(t)\left(\bar{M}_{j}+\varepsilon\right)^{\beta_{i j}}-b_{i i}(t) u_{i}\left(t-\tau_{i i}(t)\right)\right) \\
\geq & \beta_{i i} u_{i}(t)\left(r_{i}^{l}-\sum_{j=1}^{n} a_{i j}^{u}\left(\bar{M}_{j}+\varepsilon\right)^{\alpha_{i j}}\right. \\
& \left.-\sum_{j=1, j \neq i}^{n} b_{i j}^{u}\left(\bar{M}_{j}+\varepsilon\right)^{\beta_{i j}}-b_{i i}^{u}(t) u_{i}\left(t-\tau_{i i}(t)\right)\right) .
\end{aligned}
$$

Note that $\frac{r_{i}^{u}}{b_{i i}^{l}} \leq \bar{M}_{i}^{\beta_{i i}}$ implies that

$$
\begin{aligned}
\Gamma_{i \varepsilon} & =r_{i}^{l}-\sum_{j=1}^{n} a_{i j}^{u}\left(\bar{M}_{j}+\varepsilon\right)^{\alpha_{i j}}-\sum_{j=1}^{n} b_{i j}^{u}\left(\bar{M}_{j}+\varepsilon\right)^{\beta_{i j}} \\
& \leq r_{i}^{l}-b_{i i}^{u}\left(\bar{M}_{i}+\varepsilon\right)^{\beta_{i i}} \\
& \leq r_{i}^{u}-b_{i i}^{l}\left(\bar{M}_{i}+\varepsilon\right)^{\beta_{i i}} \leq 0 .
\end{aligned}
$$

Now we consider the following two cases. 
Case (i). If $\Gamma_{i \varepsilon}=0$, then for $t \geq T_{4}^{*}+\tau$, from Lemma 2.1 and (2.38) it follows that

$$
\dot{u}_{i}(t)=0 \text {, }
$$

this implies that $\lim _{t \rightarrow+\infty} u_{i}(t)=: \rho_{i}<\frac{r_{i}^{l}}{b_{i i}^{u}}$, then there exists $T_{5}^{*}>T_{4}^{*}$ such that for $t \geq T_{5}^{*}$

$$
u_{i}(t) \leq \rho_{i}+\frac{r_{i}^{l} / b_{i i}^{u}-\rho_{i}}{2}<\frac{r_{i}^{l}}{b_{i i}^{u}}<\bar{M}_{i}^{\beta_{i i}}
$$

From the $i$ th equation of system (1.4) and (2.40) if follows that

$$
\begin{aligned}
\dot{u}_{i}(t) & \geq \beta_{i i} u_{i}(t)\left(r_{i}^{l}-\sum_{j=1}^{n} a_{i j}^{u}\left(\frac{r_{j}^{l} / b_{j j}^{u}+\rho_{j}}{2}\right)^{\alpha_{i j}}-\sum_{j=1}^{n} b_{i j}^{u}\left(\frac{r_{j}^{l} / b_{j j}^{u}+\rho_{j}}{2}\right)^{\beta_{i j}}\right) \\
& >\beta_{i i} u_{i}(t) \Gamma_{i \varepsilon}=0, \quad t \geq T_{5}^{*}+\tau .
\end{aligned}
$$

Thus

$$
\begin{aligned}
u_{i}(t) \geq & u_{i}\left(T_{5}^{*}+\tau\right) \exp \left[\beta _ { i i } \left(r_{i}^{l}-\sum_{j=1}^{n} a_{i j}^{u}\left(\frac{r_{j}^{l} / b_{j j}^{u}+\rho_{j}}{2}\right)^{\alpha_{i j}}\right.\right. \\
& \left.\left.-\sum_{j=1}^{n} b_{i j}^{u}\left(\frac{r_{j}^{l} / b_{j j}^{u}+\rho_{j}}{2}\right)^{\beta_{i j}}\right)\left(t-\left(T_{5}^{*}+\tau\right)\right)\right],
\end{aligned}
$$

then we have $u_{i}(t) \rightarrow+\infty$ as $t \rightarrow+\infty$, which is in contradiction with $u_{i}(t) \leq\left(\bar{M}_{i}+\varepsilon\right)^{\beta_{i i}}$. Hence we have $\lim _{t \rightarrow+\infty} u_{i}(t) \geq \frac{r_{i}^{l}}{b_{i i}^{u}}$, which implies that there exists $T_{5}^{\prime}$ such that $u_{i}(t) \geq \frac{r_{i}^{l}}{2 b_{i i}^{u}}$ for $t \geq T_{5}^{\prime}$, that is, $x_{i}(t) \geq\left(\frac{r_{i}^{l}}{2 b_{i i}^{u}} \frac{1}{\beta_{i i}}\right.$ for $t \geq T_{5}^{\prime}$.

Case (ii). If $\Gamma_{i \varepsilon}<0$, from (2.39), for $t \geq T_{4}^{*}+\tau$, it follows that

$$
\begin{aligned}
\dot{u}_{i}(t) \geq & \beta_{i i} u_{i}(t)\left(r_{i}^{l}-\sum_{j=1}^{n} a_{i j}^{u}\left(\bar{M}_{j}+\varepsilon\right)^{\alpha_{i j}}\right. \\
& \left.-\sum_{j=1, j \neq i}^{n} b_{i j}^{u}\left(\bar{M}_{j}+\varepsilon\right)^{\beta_{i j}}-b_{i i}^{u}(t) u_{i}\left(t-\tau_{i i}(t)\right)\right) .
\end{aligned}
$$

Set

$$
\tilde{m}_{i}=\frac{r_{i}^{l}-\sum_{j=1}^{n} a_{i j}^{u}\left(\bar{M}_{j}+\varepsilon\right)^{\alpha_{i j}}-\sum_{j=1, j \neq i}^{n} b_{i j}^{u}\left(\bar{M}_{j}+\varepsilon\right)^{\beta_{i j}}}{b_{i i}^{u}}\left(1-\sigma_{i}\right),
$$

where $0<\sigma_{i}<1-\exp \left[\beta_{i i} \Gamma_{i \varepsilon} \tau\right]$.

Firstly, suppose $u_{i}(t)$ is not oscillatory about $\tilde{m}_{i}$. That is, there exists a $T_{6}^{*}>0$, for $t>T_{6}^{*}$ such that

$$
u_{i}(t)>\tilde{m}_{i}
$$

or

$$
u_{i}(t)<\tilde{m}_{i}
$$


If (2.44) holds, then our aim is obtained. Suppose (2.45) holds, then for $t \geq T_{6}^{*}+\tau$, we obtain

$$
\dot{u}_{i}(t) \geq \beta_{i i} \sigma_{i}\left(r_{i}^{l}-\sum_{j=1}^{n} a_{i j}^{u}\left(\bar{M}_{j}+\varepsilon\right)^{\alpha_{i j}}-\sum_{j=1, j \neq i}^{n} b_{i j}^{u}\left(\bar{M}_{j}+\varepsilon\right)^{\beta_{i j}}\right) u_{i}(t),
$$

thus there must exist $T_{6}^{\prime}>T_{6}^{*}+\tau$ such that $u_{i}(t)>\tilde{m}_{i}$ for $t>T_{6}^{\prime}$, which is a contradiction. Hence, (2.45) could not hold. Secondly now assume that $u_{i}(t)$ is oscillatory about $\tilde{m}_{i}$ for $t \geq T_{4}^{*}+\tau$, that is, there exists a time sequence $\left\{t_{n}\right\}$ such that $\tau<t_{i}<t_{2}<\cdots<t_{n}<\cdots$ is a sequence of zeros of $u_{i}\left(t_{n}\right)-\tilde{m}_{i}$ with $\lim _{n \rightarrow \infty} t_{n}=+\infty$ and $u_{i}\left(t_{n}\right)=\tilde{m}_{i}$. Set $\hat{t}_{n}$ to be a point where $u_{i}(t)$ attends its minimum in $\left(t_{n}, t_{n+1}\right)$. Thus, we get $u_{i}\left(\hat{t}_{n}\right) \leq u_{i}\left(t_{n}\right)=\tilde{m}_{i}$. Then it follows from (2.43) that

$$
\begin{aligned}
0= & \left.\dot{u}_{i}(t)\right|_{t=\hat{t}_{n}} \geq \beta_{i i} u_{i}\left(\hat{t}_{n}\right)\left(r_{i}^{l}-\sum_{j=1}^{n} a_{i j}^{u}\left(\bar{M}_{j}+\varepsilon\right)^{\alpha_{i j}}\right. \\
& \left.-\sum_{j=1, j \neq i}^{n} b_{i j}^{u}\left(\bar{M}_{j}+\varepsilon\right)^{\beta_{i j}}-b_{i i}^{u} u_{i}\left(\hat{t}_{n}-\tau_{i i}\left(\hat{t}_{n}\right)\right)\right),
\end{aligned}
$$

which implies that

$$
u_{i}\left(\hat{t}_{n}-\tau_{i i}\left(\hat{t}_{n}\right)\right) \geq \frac{r_{i}^{l}-\sum_{j=1}^{n} a_{i j}^{u}\left(\bar{M}_{j}+\varepsilon\right)^{\alpha_{i j}}-\sum_{j=1, j \neq i}^{n} b_{i j}^{u}\left(\bar{M}_{j}+\varepsilon\right)^{\beta_{i j}}}{b_{i i}^{u}} .
$$

Integrating (2.43) on the interval $\left[\hat{t}_{n}-\tau_{i i}\left(\hat{t}_{n}\right), \hat{t}_{n}\right]$, we have

$$
\begin{aligned}
\ln \frac{u_{i}\left(\hat{t}_{n}\right)}{u_{i}\left(\hat{t}_{n}-\tau_{i i}\left(\hat{t}_{n}\right)\right)} \geq & \int_{\hat{t}_{n}-\tau_{i i}\left(\hat{t}_{n}\right)}^{\hat{t}_{n}} \beta_{i i}\left(r_{i}^{l}-\sum_{j=1}^{n} a_{i j}^{u}\left(\bar{M}_{j}+\varepsilon\right)^{\alpha_{i j}}\right. \\
& \left.-\sum_{j=1, j \neq i}^{n} b_{i j}^{u}\left(\bar{M}_{j}+\varepsilon\right)^{\beta_{i j}}-b_{i i}^{u}(t) u_{i}\left(t-\tau_{i i}(t)\right)\right) d t \\
\geq & \int_{\hat{t}_{n}-\tau_{i i}\left(\hat{t}_{n}\right)}^{\hat{t}_{n}} \beta_{i i} \Gamma_{i \varepsilon} d t \\
= & \beta_{i i} \Gamma_{i \varepsilon} \tau_{i i}\left(\hat{t}_{n}\right) .
\end{aligned}
$$

From (2.47) and (2.48) we get

$$
u_{i}\left(\hat{t}_{n}\right) \geq \frac{r_{i}^{l}-\sum_{j=1}^{n} a_{i j}^{u}\left(\bar{M}_{j}+\varepsilon\right)^{\alpha_{i j}}-\sum_{j=1, j \neq i}^{n} b_{i j}^{u}\left(\bar{M}_{j}+\varepsilon\right)^{\beta_{i j}}}{b_{i i}^{u}} \exp \left[\beta_{i i} \Gamma_{i \varepsilon} \tau\right]
$$

Since $u_{i}\left(\hat{t}_{n}\right)$ is an arbitrary local minimum of $u_{i}(t)$, we might find there exists a $l_{i} \leq \tilde{m}_{i}$ such that $u_{i}(t) \geq l_{i}$ for all $t \geq T_{7}^{*}$. Thus, we have

$$
\lim _{t \rightarrow+\infty} \inf u_{i}(t) \geq l_{i}
$$


where

$$
l_{i} \leq\left\{\left(r_{i}^{l}-\sum_{j=1}^{n} a_{i j}^{u} \bar{M}_{j}^{\alpha_{i j}}-\sum_{j=1, j \neq i}^{n} b_{i j}^{u} \bar{M}_{j}^{\beta_{i j}}\right) / b_{i i}^{u}, i=1,2, \ldots, m\right\} .
$$

Take $\bar{m}_{i}=\min _{1 \leq i \leq n}\left\{\left(\frac{r_{i}^{l}}{2 b_{i}^{u}}\right)^{\frac{1}{\beta_{i i}}}, l_{i}^{\frac{1}{\beta_{i i}}}\right\}$, we obtain

$$
\lim _{t \rightarrow+\infty} \inf x_{i}(t) \geq \bar{m}_{i}
$$

From the condition $\left(\mathrm{H}_{6}\right)$ of Theorem 2.2, we could choose $\varepsilon>0$ small enough such that

$$
\begin{aligned}
-r_{i}^{u} & +\sum_{j=1}^{m} a_{i j}^{l}\left(m_{j}-\varepsilon\right)^{\alpha_{i j}}+\sum_{j=1}^{m} b_{i j}^{l}\left(m_{j}-\varepsilon\right)^{\beta_{i j}} \\
& -\sum_{j=m+1, j \neq i}^{n} a_{i j}^{u}\left(M_{j}+\varepsilon\right)^{\alpha_{i j}}-\sum_{j=m+1}^{n} b_{i j}^{u}\left(M_{j}+\varepsilon\right)^{\beta_{i j}} \geq 0,
\end{aligned}
$$

thus, for $\varepsilon>0$ it satisfies (2.50), and it follows from (2.49) that there exists large enough $T_{8}^{*}>T_{7}^{*}$ such that for all $i=1,2, \ldots, m$ and $t \geq T_{8}^{*}$

$$
x_{i}(t) \geq \bar{m}_{i}-\varepsilon .
$$

For $i=m+1, \ldots, n$ and $t \geq T_{8}^{*}+\tau$, by using (2.51), from the $i$ th equation of system (1.4), one has

$$
\begin{gathered}
\dot{x}_{i}(t) \geq x_{i}(t)\left(-r_{i}^{u}+\sum_{j=1}^{m} a_{i j}^{l}\left(m_{j}-\varepsilon\right)^{\alpha_{i j}}+\sum_{j=1}^{m} b_{i j}^{l}\left(m_{j}-\varepsilon\right)^{\beta_{i j}}\right. \\
\left.-\sum_{j=m+1}^{n} a_{i j}^{u}\left(M_{j}+\varepsilon\right)^{\alpha_{i j}}-\sum_{j=m+1}^{n} b_{i j}^{u}\left(M_{j}+\varepsilon\right)^{\beta_{i j}}\right)
\end{gathered}
$$

and

$$
\begin{aligned}
\dot{x}_{i}(t) \geq & x_{i}(t)\left(-r_{i}(t)+\sum_{j=1}^{m} a_{i j}(t)\left(m_{j}-\varepsilon\right)^{\alpha_{i j}}+\sum_{j=1}^{m} b_{i j}(t)\left(m_{j}-\varepsilon\right)^{\beta_{i j}}\right. \\
& \left.-\sum_{j=m+1}^{n} a_{i j}(t)\left(M_{j}+\varepsilon\right)^{\alpha_{i j}}-\sum_{j=m+1, j \neq i}^{n} b_{i j}(t)\left(M_{j}+\varepsilon\right)^{\beta_{i j}}-b_{i i}(t) x_{i}^{\beta_{i i}}\left(k-\tau_{i i}(t)\right)\right) .
\end{aligned}
$$

From (2.52) and (2.53), similar to the argument of (2.34) and (2.35), we also have

$$
\lim _{t \rightarrow+\infty} \inf x_{i}(t) \geq \bar{m}_{i}, \quad i=m+1, \ldots, n
$$

where

$$
\bar{m}_{i}=\min _{1 \leq i \leq n}\left\{\left(\frac{-r_{i}^{u}+\sum_{j=1}^{m} a_{i j}^{l} m_{j}^{\alpha_{i j}}+\sum_{j=1}^{m} b_{i j}^{l} m_{j}^{\beta_{i j}}}{2 b_{i}^{u}}\right)^{\frac{1}{\beta_{i i}}}, l_{i}^{\frac{1}{\beta_{i i}}}\right\},
$$




$$
\begin{aligned}
& l_{i} \leq\left\{\nabla_{i} / b_{i i}^{u}, i=m+1, \ldots, n\right\}, \\
& \nabla_{i}=-r_{i}^{u}+\sum_{j=1}^{m} a_{i j}^{l} m_{j}^{\alpha_{i j}}+\sum_{j=1}^{n} b_{i j}^{l} m_{j}^{\beta_{i j}}-\sum_{j=m+1}^{n} a_{i j}^{u} M_{j}^{\alpha_{i j}}-\sum_{j=m+1, j \neq i}^{n} b_{i j}^{u} M_{j}^{\beta_{i j}} .
\end{aligned}
$$

Take $\bar{M}=\max _{1 \leq i \leq n}\left\{\bar{M}_{i}\right\}, \bar{m}=\min _{1 \leq i \leq n}\left\{\bar{m}_{i}\right\}$, we have

$$
\bar{m} \leq \liminf _{t \rightarrow+\infty} x_{i}(t) \leq \limsup _{t \rightarrow+\infty} x_{i}(t) \leq \bar{M}, \quad i=1,2, \ldots, n .
$$

This completes the proof of Theorem 2.2.

\section{Concluding remarks}

In this paper, we study a Gilpin-Ayala predator-prey model with time-dependent delay with $m$ preys and $(n-m)$ predators. In this system, the competition among the predator species and among the prey species are simultaneously considered. The system (1.4) can be seen as the modification of the traditional Lotka-Volterra prey-competition system. Some new and interesting sufficient conditions are obtained for the permanence of the system (1.4). In [7], under the assumption that the coefficient of the density-dependent term $a_{i i}(t)$ must be positive, by using a new differential inequality, two sets of sufficient conditions on the permanence of the system (1.4) are obtained. However, in this paper we allow the coefficient to be zero; therefore the study of the permanence of the population becomes technically more difficult. Our results are different from the existing ones such as those of Huang [7]. In some sense, our results supplement those obtained by Chen et al. [6], generalize the results in [7], and have further application on the population dynamics.

Competing interests

The authors declare that they have no competing interests.

Authors' contributions

HL carried out the main part of this article, GY corrected the main theorems. All authors read and approved the final manuscript.

\section{Author details}

${ }^{1}$ School of Mathematics and Quantitative Economics, Dongbei University of Finance and Economics, Jianshan street, Dalian, China. ${ }^{2}$ School of Economics, Huazhong University of Science and Technology, Luoyu road, Wuhan, 430074, China.

\section{Acknowledgements}

The authors are grateful to the editor and two referees for a number of helpful suggestions, which have greatly improved our original manuscript. This research is supported by MOE (Ministry of Education in China) Youth Foundation Project of Humanities and Social Sciences (No. 13YJC790185), National Natural Science Foundation of China under Grants (Nos. 71171035, 71471030), China Postdoctoral Science Foundation (Nos. 2012M511598, 2013T60710), Liaoning Educational Committee Special Project of Humanities and Social Science Key Research Base (No. ZJ2013039), Liaoning Education Science Twelfth Five Year Plan (No. JG14DB152), General Project for Scientific Research of Liaoning Educational Committee (L2014458), and Dongbei University of Finance and Economics Project of Excellent Scientific Research Innovative Talents (No. DUFE2014R11).

Received: 10 October 2014 Accepted: 28 December 2014 Published online: 01 April 2015

\section{References}

1. Berryman, AA: The origins and evolution of predator-prey theory. Ecology 73, 1530-1535 (1992)

2. Volterra, V: Variazioni e fluttuazioni del numero d'individui in specie d'animali conviventi. Mem. R. Accad. Naz. Dei Lincei 2, 31-113 (1926)

3. Lotka, A: Elements of Physical Biology. Williams \& Wilkins, Baltimore (1924)

4. Ayala, FJ, Gilpin, ME, Eherenfeld, JG: Competition between species: theoretical models and experimental tests. Theor. Popul. Biol. 4, 331-356 (1973)

5. Li, CR, Lu, SJ: The qualitative analysis of $\mathrm{N}$-species periodic coefficient, nonlinear relation, prey-competition systems. Appl. Math. J. Chin. Univ. Ser. A 12, 147-156 (1997) 
6. Chen, FD, Xie, XD, Shi, JL: Existence, uniqueness and stability of positive periodic solution for a nonlinear prey-competition model with delays. J. Comput. Appl. Math. 194, 368-387 (2006)

7. Huang, AM: Permanence of a nonlinear prey-competition model with delays. Appl. Math. Comput. 197, 372-381 (2008)

8. Tineo, A, Alvarz, C: A different consideration about the globally asymptotically stable solution of the periodic n-competition species problem. J. Math. Anal. Appl. 159, 44-55 (1991)

9. Meng, XM, Chen, LS: Periodic solution and almost periodic solution for a nonautonomous Lotka-Volterra dispersal system with infinite delay. J. Math. Anal. Appl. 339, 125-145 (2008)

10. Meng, XM, Chen, LS: Almost periodic solution of non-autonomous Lotka-Volterra predator-prey dispersal system with delays. J. Theor. Biol. 243, 562-574 (2006)

11. Ding, XH, Lu, C, Liu, MZ: Periodic solutions for a semi-ratio-dependent predator-prey system with nonmonotonic functional response and time delay. Nonlinear Anal., Real World Appl. 9, 762-775 (2008)

12. Ding, XH, Lu, C: Existence of positive periodic solution for ratio-dependent $\mathrm{N}$-species difference system. Appl. Math. Model. 33, 2748-2756 (2008)

13. Xu, R, Chaplain, MAJ: Persistence and global stability in a delayed predator-prey system with Michaelis-Menten type functional response. Appl. Math. Comput. 130, 441-455 (2002)

14. Zhao, JD, Chen, WC: The qualitative analysis of $\mathrm{N}$-species nonlinear prey-competition systems. Appl. Math. Comput. $149,567-576(2004)$

15. Xu, CJ, Wu, YS: Dynamics in a Lotka-Volterra predator-prey model with time-varying delays. Abstr. Appl. Anal. 2013, Article ID 956703 (2013)

16. Yang, P, Xu, R: Global attractivity of the periodic Lotka-Volterra system. J. Math. Anal. Appl. 233, 221-232 (1999)

17. Wang, WD, Chen, LS, Lu, ZY: Global stability of a competition model with periodic coefficients and time delay. Can. Appl. Math. Q. 3, 365-378 (1995)

18. Muroya, Y: Persistence and global stability in Lotka-Volterra delay differential systems. Appl. Math. Lett. 17, 795-800 (2004)

19. Wang, W, Lu, Z: Global stability of discrete models of Lotka-Volterra type. Nonlinear Anal., Theory Methods Appl. 35 , 1019-1030 (1999)

20. Chen, FD, Li, Z, Xie, XD: Permanence of a nonlinear integro-differential prey-competition model with infinite delays. Commun. Nonlinear Sci. Numer. Simul. 13, 2290-2297 (2008)

21. Chen, FD, Xie, XD: Periodicity and stability of a nonlinear periodic integro-differential prey-competition model with infinite delays. Commun. Nonlinear Sci. Numer. Simul. 12, 876-885 (2007)

22. De la Sen, M: Stability of impulsive time-varying systems and compactness of the operators mapping the input space into the state and output spaces. J. Math. Anal. Appl. 321, 621-650 (2006)

23. Lu, HY, Wang, WG: Dynamics of a delayed discrete semi-ratio-dependent predator-prey system with Holling type IV functional response. Adv. Differ. Equ. (2011). doi:10.1186/1687-1847-2011-7

24. Lu, HY, Wang, WG: Dynamics of a nonautonomous Leslie-Gower type food chain model with delay. Discrete Dyn. Nat. Soc. 2011, Article ID 308279 (2011)

25. Yu, G, Lu, HY: Permanence and almost periodic solutions of a discrete ratio-dependent Leslie system with time delays and feedback controls. Abstr. Appl. Anal. 2012, Article ID 358594 (2012)

26. Gilpin, ME, Ayala, FJ: Global models of growth and competition. Proc. Natl. Acad. Sci. USA 70, 3590-3593 (1973)

\section{Submit your manuscript to a SpringerOpen ${ }^{\ominus}$ journal and benefit from:}

- Convenient online submission

Rigorous peer review

- Immediate publication on acceptance

Open access: articles freely available online

- High visibility within the field

- Retaining the copyright to your article 